\title{
Use of Harriman's construction in determining molecular equilibrium states
}

\author{
Roman F. Nalewajski
}

Received: 22 July 2012 / Accepted: 12 September 2012 / Published online: 28 September 2012 (C) The Author(s) 2012. This article is published with open access at Springerlink.com

\begin{abstract}
Information-theoretic description of the electron probabilities and currents in molecules is extended to cover the complex amplitudes (wave functions) of quantum mechanics. The classical information measures of Fisher and Shannon, due to the probability/density distributions themselves, are supplemented by the nonclassical terms generated by the wave-function phase or the associated probability current. The previous one-electron development in such an entropic perspective on the molecular electronic structure is extended to cover $N$-electron states by adopting the Harrimantype framework of equidensity orbitals. This analysis emphasizes the phase part of electronic states, which generates the probability-current density and the associated non-classical entropy contributions, which allow one to distinguish the information content of states generating the same electron density and differing in their current composition. A complementary character of the Fisher and Shannon information measures is explored in the associated vertical (density-constrained) information principles, for determining the equilibrium state corresponding to the fixed ground-state electron density. It is argued that the lowest "thermodynamic" state generally differs from the true ground state of the system, by exhibiting the space-dependent phase and hence also the non-vanishing probability current, linked to the system electron distribution.
\end{abstract}

Keywords Density-constrained principles · Electronic structure theory · Entropic principles for molecules · Harriman-Zumbach-Maschke construction · Nonclassical information measures · Information equilibrium states

R. F. Nalewajski $(\varangle)$

Department of Theoretical Chemistry, Jagiellonian University, R. Ingardena 3, 30-060,

Cracow, Poland

e-mail: nalewajs@chemia.uj.edu.pl 


\section{Introduction}

The classical Fisher (local) [1] or Shannon (global) [2] information descriptors of the particle spatial distribution are determined solely by the probability aspect of the complex wave function. The associated phase facet of the latter, which generates the probability current, was shown to give rise to additional, nonclassical terms in the quantum extensions of these gradient (Fisher) and logarithmic (Shannon) measures of the information content in the complex quantum states, e.g., [3-8]. In the one-electron system the densities of such phase-related information probes have been shown to be proportional to the square of the local probability current [6] or the phase function itself $[7,8]$, respectively. These generalized, quantum measures allow one to distinguish the amount of information in states exhibiting the same particle density and differing in their phase (current) composition. ${ }^{1}$

This phase side of the molecular electronic structure reflects its "entropic" aspect, which still remains largely unexplored. It has been recently demonstrated [8] for the one-electron system that the lowest equilibrium state corresponding to the maximum (quantum) entropy, which gives rise to the ground-state particle distribution, is characterized by the space-dependent (local) phase linked to the ground-state probability density itself. Therefore, it also exhibits a nonvanishing probability current, and thus differs from the lowest eigenstate of the system Hamiltonian,

$$
\hat{\mathrm{H}}(N)=\hat{\mathrm{V}}_{n e}(N)+\left[\hat{\mathrm{T}}(N)+\hat{\mathrm{V}}_{e e}(N)\right] \equiv \sum_{i=1}^{N} v(i)+\hat{\mathrm{F}}(N),
$$

in which the particle current identically vanishes. Here, $v(\boldsymbol{r})$ stands for the external potential due to the system fixed nuclei, and $\hat{\mathrm{F}}(N)$ combines the electron kinetic $(\hat{\mathrm{T}})$ and repulsion $\left(\hat{\mathrm{V}}_{e e}\right)$ energy operators.

In the past such an "entropic" interpretation has been attributed to the density-constrained principles $[9,10]$ in the modern Density Functional Theory (DFT) [11-15], in the so called vertical (entropic) variational searches performed for the specified electron density $\rho$, e.g., in Levy's [10] constrained-search construction of the universal part of the energy density functional,

$$
F[\rho]=T[\rho]+V_{e e}[\rho]=\inf _{\Psi \rightarrow \rho}\langle\Psi|\hat{\mathrm{F}}| \Psi\rangle .
$$

In this variational procedure one searches over the wave functions $\Psi(N)$ of $N$ electrons, which yield the given electron density $\rho$, symbolically denoted by $\Psi \rightarrow \rho$, and calculates the $v$-independent part $F[\rho]$ of the density functional for the system electronic energy,

$$
E_{v}[\rho]=F[\rho]+\int v(\boldsymbol{r}) \rho(\boldsymbol{r}) d \boldsymbol{r}
$$

\footnotetext{
1 Here $A, \boldsymbol{A}$, and $\mathbf{A}$ denote the scalar quantity, row vector and a square/rectangular matrix, respectively. The logarithm of the information measure is taken to an arbitrary but fixed base: $\log =\log _{2}$ corresponds to information measured in bits (binary digits), while $\log =\ln$ expresses the amount of information in nats (natural units): 1 nat $=1.44$ bits
} 
as the lowest value (infimum) of the expectation value of $\hat{\mathrm{F}}(N)$. When this search is performed for the fixed ground-state density, it also implies the fixed value of the system electronic energy, by the first Hohenberg-Kohn (HK) theorem [11] of DFT. This feature is reminiscent of the familiar thermodynamic criterion for the equilibrium state formulated in the entropy representation [16].

Therefore, by analogy to the maximum-entropy principle in the ordinary phenomenological thermodynamics [16], for constant internal energy, this DFT construction has been also regarded as being "entropic" in character [9]. The familiar variational principle for determining the ground state wave function, involving a search for the minimum of the system energy, can be thus interpreted as the DFT optimization over all admissible densities, in accordance with the second HK theorem [11], which involves the "internal" (entropic) search over functions of $N$ fermions that yield the current trial density:

$$
\min _{\Psi}\langle\Psi|\hat{\mathrm{H}}| \Psi\rangle=\min _{\rho} E_{v}[\rho]=\min _{\rho}\left\{\int v(\boldsymbol{r}) \rho(\boldsymbol{r}) d \boldsymbol{r}+\inf _{\Psi \rightarrow \rho}\langle\Psi|\hat{\mathrm{F}}| \Psi\rangle\right\} .
$$

A similar idea of the space-dependent phase is adopted in constructions of antisymmetric states that generate the specified electron density [17-22]. It is required to ensure both the orthonormality and completeness of the underlying orbital basis for constructing the complete set of Slater determinants, which are capable of exactly representing any $N$-electron state for the prescribed electron distribution. The particular choice of the equidensity orthonormal orbitals defining the Slater determinant that yields the given electron density $\rho(\boldsymbol{r})$ in such a vertical (density-constrained) search has been proposed by Harriman [17] on the basis of the pioneering works by Macke [18] and Gilbert [19]. His construction explicitly demonstrates the (pure-state) $N$-representability of any (non-negative) distribution $\rho(\boldsymbol{r})$ that integrates to $N$ electrons. Alternative constructions and extensions have also been suggested, e.g., [20-22].

In this analysis we focus on the use of this type of constructing the molecular wave functions in probing the physical implications of the lowest equilibrium ("thermodynamic") state [8] in the molecular quantum mechanics. It results from the equivalent principles of the maximum Shannon entropy or the minimum Fisher-information, using the non-classical measures of the information content of the system wave function $[7,8]$. Although in the one-electron case the molecular (maximum-entropy) equilibrium exhibits the same energy as the true ground state, it also involves the local, real-valued phase function related to the probability distribution, and hence the nonvanishing probability current. This is contrary to the non-degenerate eigenstate of the Hamiltonian, for which the current identically vanishes. In what follows we extend the previous one-electron analysis to the entropy/information equilibria in general $N$-electron systems by adopting the equidensity orbital framework of the HarrimanZumbach-Maschke construction.

\section{Harriman construction}

Let us briefly outline the main idea behind the Gilbert-Harriman prescription for constructing the $N$-electron Slater determinant that yields the prescribed overall electron 
density $\rho(\boldsymbol{r})=\Sigma_{k} \rho_{k}(\boldsymbol{r})=N \rho_{k}(\boldsymbol{r})$, from orbitals generating equal density contributions $\left\{\rho_{k}(\boldsymbol{r})=\rho(\boldsymbol{r}) / N\right\}$. As in the original presentation [17] we first assume, for reasons of simplicity, the one-dimensional case of the nonnegative electron distribution $\rho(x)$ with $\int_{-\infty}^{\infty} \rho(x) d x=N$. One then introduces the auxiliary, strictly monotonically increasing function $f(x)$, defined by the differential equation,

$$
\frac{d f(x)}{d x}=\frac{2 \pi}{N} \rho(x),
$$

or the associated indefinite integral

$$
f(x)=\frac{2 \pi}{N} \int_{-\infty}^{x} \rho(y) d y .
$$

It defines the single-particle states (orbitals), of the plane-wave type, each corresponding to the same piece of the overall electron density $\left\{\rho_{k}(x)=\rho(x) / N\right\}$,

$$
\varphi_{k}(x)=\rho_{k}(x)^{1 / 2} \exp \{\mathrm{i}[k f(x)+\phi(x)]\} \equiv \rho_{k}(x)^{1 / 2} \exp \left\{\mathrm{i} \Phi_{k}(x)\right],
$$

where $\phi(x)$ stands for an arbitrary real-valued phase function. One readily demonstrates using Eq. (5) this basis set orthogonality and completeness [14,17], e.g.,

$$
\begin{aligned}
\int_{-\infty}^{\infty} \varphi_{k^{\prime}}^{*}(x) \varphi_{k}(x) d x & =\int_{-\infty}^{\infty} \mathrm{e}^{\mathrm{i}\left(k-k^{\prime}\right) f(x)} \frac{\rho(x)}{N} d x \\
& =\frac{1}{2 \pi} \int_{-\infty}^{\infty} \mathrm{e}^{\mathrm{i}\left(k-k^{\prime}\right) f(x)} \frac{d f}{d x} d x=\frac{1}{2 \pi} \int_{-\infty}^{\infty} \mathrm{e}^{\mathrm{i}\left(k-k^{\prime}\right) f} d f=\delta\left(k-k^{\prime}\right) .
\end{aligned}
$$

Therefore, the Slater determinants build from a given selection of $N$ equidensity orbitals,

$$
\left\{\Psi_{k_{1}, k_{2}, \ldots, k_{N}}=(1 / \sqrt{N !}) \operatorname{det}\left[\varphi_{k_{1}}, \varphi_{k_{2}}, \ldots, \varphi_{k_{N}}\right], k_{i} \neq k_{j} \quad \text { for } i \neq j\right\}
$$

constitute the complete orthonormal system of $N$-particle functions, each corresponding to the prescribed electron density $\rho(x)=\Sigma_{k}\left|\varphi_{k}(x)\right|^{2}=N \rho_{k}(x)$, capable of representing any $N$-electron molecular state for this specific electron distribution.

These ideas have been extended into three dimensions [21] and molecular subsystems [22]. The three-dimensional case is also based on the plane-wave type orbitals,

$$
\begin{aligned}
\varphi_{\boldsymbol{k}}(\boldsymbol{r}) & =[\rho(\boldsymbol{r}) / N]^{1 / 2} \exp \{\mathrm{i}[\boldsymbol{k} \cdot \boldsymbol{f}(\boldsymbol{r})+\phi(\boldsymbol{r})]\} \equiv[\rho(\boldsymbol{r}) / N]^{1 / 2} \exp \left[\mathrm{i} \Phi_{\boldsymbol{k}}(\boldsymbol{r})\right] \\
& \equiv\left\{[\rho(\boldsymbol{r}) / N]^{1 / 2} \exp \left[\mathrm{i} F_{\boldsymbol{k}}(\boldsymbol{r})\right]\right\} \exp [\mathrm{i} \phi(\boldsymbol{r})] \equiv R_{\boldsymbol{k}}(\boldsymbol{r}) \exp [\mathrm{i} \phi(\boldsymbol{r})],
\end{aligned}
$$


where $\boldsymbol{k}=\left(k_{x}, k_{y}, k_{z}\right)$ and $\boldsymbol{f}=\left(f_{1}, f_{2}, f_{3}\right)=\boldsymbol{f}[\rho]$ with the density-related Jacobian determinant,

$$
\frac{\partial \boldsymbol{f}}{\partial \boldsymbol{r}}=\frac{(2 \pi)^{3}}{N} \rho(\boldsymbol{r})
$$

reflecting the associated transformation between the volume elements:

$$
d \boldsymbol{f}=d \boldsymbol{r} \frac{\partial \boldsymbol{f}}{\partial \boldsymbol{r}}
$$

As explicitly proposed by Zumbach and Maschke [21]:

$$
\begin{aligned}
f_{1}(x, y, z) & =2 \pi \frac{\int_{-\infty}^{x} d x^{\prime} \rho\left(x^{\prime}, y, z\right)}{\int_{-\infty}^{\infty} d x^{\prime} \rho\left(x^{\prime}, y, z\right)}, \\
f_{2}(y, z) & =2 \pi \frac{\int_{-\infty}^{y} d y^{\prime} \int_{-\infty}^{\infty} d x^{\prime} \rho\left(x^{\prime}, y^{\prime}, z\right)}{\int_{-\infty}^{\infty} d y^{\prime} \int_{-\infty}^{\infty} d x^{\prime} \rho\left(x^{\prime}, y^{\prime}, z\right)} \\
f_{3}(z) & =\frac{2 \pi}{N} \int_{-\infty}^{z} d z^{\prime} \int_{-\infty}^{\infty} d y^{\prime} \int_{-\infty}^{\infty} d x^{\prime} \rho\left(x^{\prime}, y^{\prime}, z^{\prime}\right) .
\end{aligned}
$$

Therefore, this construction involves the transformation of the physical space into itself: for any $\boldsymbol{r}=(x, y, z) \in \mathfrak{R}^{3}$ one defines the (invertible) density-dependent transformation of $\boldsymbol{r}$ into the new vector $\boldsymbol{f}(\boldsymbol{r}) \in \mathfrak{R}^{3}$, with the orbital orthogonality relation of Eq. (8) being now generalized into its three-dimensional analog:

$$
\begin{aligned}
\int_{-\infty}^{\infty} \varphi_{\boldsymbol{k}^{\prime}}^{*}(\boldsymbol{r}) \varphi_{\boldsymbol{k}}(\boldsymbol{r}) d \boldsymbol{r} & =\int_{-\infty}^{\infty} \mathrm{e}^{\mathrm{i}\left(\boldsymbol{k}-\boldsymbol{k}^{\prime}\right) \cdot \boldsymbol{f}(\boldsymbol{r})} \frac{\rho(\boldsymbol{r})}{N} d \boldsymbol{r} \\
& =\frac{1}{(2 \pi)^{3}} \int_{-\infty}^{\infty} \mathrm{e}^{\mathrm{i}\left(\boldsymbol{k}-\boldsymbol{k}^{\prime}\right) \cdot \boldsymbol{f}(\boldsymbol{r})} \frac{\partial \boldsymbol{f}}{\partial \boldsymbol{r}} d \boldsymbol{r} \\
& =\frac{1}{(2 \pi)^{3}} \int_{-\infty}^{\infty} \mathrm{e}^{\mathrm{i}\left(\boldsymbol{k}-\boldsymbol{k}^{\prime}\right) \cdot \boldsymbol{f}} d \boldsymbol{f}=\delta\left(\boldsymbol{k}-\boldsymbol{k}^{\prime}\right) .
\end{aligned}
$$

The overall phase factor $\exp \left[\mathrm{i} \Phi_{k}(\boldsymbol{r})\right]$ of these equidensity orbitals [see Eq. (10)] involves the orbital-specific orthogonality (geometric) part exp $[\mathrm{i} \boldsymbol{k} \cdot \boldsymbol{f}(\boldsymbol{r})] \equiv \exp \left[\mathrm{i} F_{\boldsymbol{k}}(\boldsymbol{r})\right]$, which enforces the independence of these one-particle states, and the "thermodynamic" (physical) factor $\exp [\mathrm{i} \phi(\boldsymbol{r})]$ due to an arbitrary phase $\phi(\boldsymbol{r})$, common to all orbitals,

$$
\Phi_{k}(\boldsymbol{r})=F_{k}(\boldsymbol{r})+\phi(\boldsymbol{r})
$$


The latter has to be determined from some additional variational principle, either energetic [12,21] or entropic in character [8]. It introduces the entropic factor

$$
\Phi(N)=\prod_{k=1}^{N} \exp \left[\mathrm{i} \phi\left(\boldsymbol{r}_{k}\right)\right]=\exp \left[\mathrm{i} \sum_{k=1}^{N} \phi\left(\boldsymbol{r}_{k}\right)\right] \equiv \exp [\mathrm{i} \phi(N)]
$$

of the overall (symmetric) phase factor in the trial $N$-electron Slater determinant,

$$
\begin{aligned}
\Psi_{\boldsymbol{k}_{1}, \boldsymbol{k}_{1}, \ldots, \boldsymbol{k}_{N}}(N) & =(1 / \sqrt{N !}) \operatorname{det}\left[\varphi_{\boldsymbol{k}_{1}}, \varphi_{\boldsymbol{k}_{2}}, \ldots, \varphi_{\boldsymbol{k}_{N}}\right] \equiv \Psi_{\mathbf{k}}(N) \\
& =(1 / \sqrt{N !}) \operatorname{det}\left[R_{\boldsymbol{k}_{1}}, R_{\boldsymbol{k}_{2}}, \ldots, R_{\boldsymbol{k}_{N}}\right] \Phi(N) \equiv R_{\mathbf{k}}(N) \Phi(N)
\end{aligned}
$$

which generates the prescribed electron density $\rho(\boldsymbol{r})$ :

$$
\left\langle\Psi_{\mathbf{k}}|\hat{\rho}(\boldsymbol{r})| \Psi_{\mathbf{k}}\right\rangle=\sum_{i=1}^{N}\left|\varphi_{\boldsymbol{k}_{i}}(\boldsymbol{r})\right|^{2}=\rho(\boldsymbol{r}), \quad \hat{\rho}(\boldsymbol{r})=\sum_{i=1}^{N} \delta\left(\boldsymbol{r}_{i}-\boldsymbol{r}\right) .
$$

This determinantal wave function also includes the symmetrical modulus part,

$$
R(N)=\prod_{k=1}^{N}\left(\frac{\rho\left(\boldsymbol{r}_{k}\right)}{N}\right)^{1 / 2}
$$

and the remaining determinant of $N$ electrons involving the transformed "planewaves" $\left\{\pi_{\boldsymbol{k}_{i}}\left(\boldsymbol{r}_{j}\right)=\exp \left(i \boldsymbol{k}_{i} \cdot \boldsymbol{f}\left(\boldsymbol{r}_{j}\right)\right\}\right.$ :

$$
\begin{aligned}
\Psi_{\mathbf{k}}(N) & =(1 / \sqrt{N !}) R(N) \Phi(N) \operatorname{det}\left[\pi_{\boldsymbol{k}_{1}}, \pi_{\boldsymbol{k}_{2}}, \ldots, \pi_{\boldsymbol{k}_{N}}\right] \\
& =R(N) \Phi(N)(1 / \sqrt{N !}) \mid \exp \left(\mathrm{i} \boldsymbol{k}_{i} \cdot \boldsymbol{f}\left(\boldsymbol{r}_{j}\right) \mid .\right.
\end{aligned}
$$

Each equidensity orbital $\phi_{\boldsymbol{k}}(\boldsymbol{r})$ generates the associated probability-current contribution

$$
\boldsymbol{j}_{\boldsymbol{k}}(\boldsymbol{r})=\left\langle\phi_{\boldsymbol{k}}|\hat{\mathbf{j}}(\boldsymbol{r})| \phi_{\boldsymbol{k}}\right\rangle=\frac{\hbar \rho(\boldsymbol{r})}{m N} \nabla \Phi_{\boldsymbol{k}}(\boldsymbol{r}) \equiv \frac{\hbar \rho(\boldsymbol{r})}{m N}[\boldsymbol{k} \nabla \cdot \boldsymbol{f}(\boldsymbol{r})+\nabla \phi(\boldsymbol{r})]
$$

the expectation value of the associated operator in the position representation:

$$
\hat{\mathbf{j}}(\boldsymbol{r})=\frac{\hbar}{2 m i}\left[\delta\left(\boldsymbol{r}^{\prime}-\boldsymbol{r}\right) \nabla_{\boldsymbol{r}^{\prime}}+\nabla_{\boldsymbol{r}^{\prime}} \delta\left(\boldsymbol{r}^{\prime}-\boldsymbol{r}\right)\right] .
$$


Hence, the overall current $\boldsymbol{j}(\boldsymbol{r})=\left\langle\Psi_{\mathbf{k}}(N)|\hat{\mathbf{j}}(\boldsymbol{r} ; N)| \Psi_{\mathbf{k}}(N)\right\rangle$ in the Slater determinant $\Psi_{\mathbf{k}}(N)$, corresponding to the $N$-electron current operator $\hat{\mathbf{j}}(\boldsymbol{r} ; N)=\sum_{l=1}^{N} \hat{\mathbf{j}}_{l}(\boldsymbol{r})$,

$$
\begin{aligned}
\boldsymbol{j}_{\mathbf{k}}(\boldsymbol{r}) & =\left\langle\Psi_{\mathbf{k}}(N)|\hat{\mathbf{j}}(\boldsymbol{r} ; N)| \Psi_{\mathbf{k}}(N)\right\rangle=\sum_{l=1}^{N} \boldsymbol{j}_{\boldsymbol{k}_{l}}(\boldsymbol{r})=\frac{\hbar \rho(\boldsymbol{r})}{m N}\left(\sum_{l=1}^{N}\left[\boldsymbol{k}_{l} \nabla \cdot \boldsymbol{f}(\boldsymbol{r})+\nabla \varphi(\boldsymbol{r})\right]\right) \\
& =\frac{\hbar}{m} \rho(\boldsymbol{r})\left(\frac{1}{N} \sum_{l=1}^{N} \boldsymbol{k}_{l}\right) \nabla \cdot \boldsymbol{f}(\boldsymbol{r})+\frac{\hbar}{m} \rho(\boldsymbol{r}) \nabla \phi(\boldsymbol{r}) \\
& \equiv \frac{\hbar}{m} \rho(\boldsymbol{r})[\boldsymbol{K} \nabla \cdot \boldsymbol{f}(\boldsymbol{r})+\nabla \phi(\boldsymbol{r})],
\end{aligned}
$$

with $\boldsymbol{K}$ denoting the average "wave-number" (reduced momentum) vector of $\Psi_{\mathbf{k}}(N)$ and $\nabla \cdot \boldsymbol{f}=\partial f_{1} / \partial x+\partial f_{2} / \partial y+\partial f_{3} / \partial z$ standing for the divergence of the densitydependent vector field $\boldsymbol{f}(\boldsymbol{r})$.

Therefore, both parts of the orbital phase-factor in Harriman's construction contribute to the probability current in such a representative trial Slater determinant. The DFT search of Eq. (4), in spirit of the second Hohenberg-Kohn (HK) theorem [11], is seen to involve the combined "external" (horizontal) variations of the system electron distribution and the "internal" (vertical) variations [9] of trial orbitals for the current (fixed) electron density.

In DFT this explicit construction of the antisymmetric wave functions of $N$ electrons corresponding to the prescribed electron density $\rho(\boldsymbol{r})$ [Eqs. (16) and (19)] has been vital for solving the familiar $N$-representability problem of DFT. It has also been used to establish reliable estimates for the density functionals and rigorous bounds for parts of the exact ground state energy, e.g., [14]. In what follows we shall explore the optimum phase functions $\left\{\Phi_{\boldsymbol{k}}(\boldsymbol{r})\right\}$ resulting from the recently discussed Extreme Information Principles (EIP), using the quantum-generalized information functionals, appropriately modified to tackle the complex probability amplitudes (wave functions) of quantum mechanics [6-8].

\section{Information principles}

We first observe that in the position representation the common phase factor does not contribute to the expectation values of the multiplicative operators, e.g., of the potential energy, but it becomes essential in determining the kinetic energy (quantum Fisher information) term [6-8] due to the probability current. It also directly affects the nonclassical (quantum) complement

$$
S^{\text {nclass. }}[p, \phi]=2 \int p(\boldsymbol{r}) \phi(\boldsymbol{r}) d \boldsymbol{r} \equiv \int p(\boldsymbol{r}) S^{\text {nclass. }}(\boldsymbol{r}) d \boldsymbol{r}
$$

of the classical Shannon entropy,

$$
S^{\text {class. }}[p]=-\int p(\boldsymbol{r}) \log p(\boldsymbol{r}) d \boldsymbol{r} \equiv \int p(\boldsymbol{r}) S^{\text {class. }}(\boldsymbol{r}) d \boldsymbol{r}
$$


in the quantum-generalized average entropy concept $[7,8]$ :

$$
S[\Psi] \equiv S[p, \Phi]=S^{\text {class. }}[p]+S^{n \text { class. }} \cdot[p, \phi] \equiv \int p(\boldsymbol{r}) S(\boldsymbol{r}) d \boldsymbol{r},
$$

where the probability distribution $p(\boldsymbol{r})=\rho(\boldsymbol{r}) / N$ represents the "shape" factor of the electron density. This nonclassical extension has been derived [7] by a natural requirement that the relation between the classical Shannon and Fisher information densities $[7,8,23]$ should also hold for their nonclassical complements.

Consider the minimum of the quantum Fisher information in state $\Psi_{\mathbf{k}}(N)$,

$$
\begin{aligned}
I\left[\Psi_{\mathbf{k}}\right] & =4 \sum_{i=1}^{N} \int\left|\nabla \varphi_{\boldsymbol{k}_{i}}(\boldsymbol{r})\right|^{2} d \boldsymbol{r}=\frac{8 m}{\hbar^{2}} T\left[\Psi_{\mathbf{k}}\right] \equiv \int p(\boldsymbol{r}) I(\boldsymbol{r}) d \boldsymbol{r} \\
& =\int p(\boldsymbol{r})\left[I^{\text {class. }}(\boldsymbol{r})+I^{\text {nclass. }}(\boldsymbol{r})\right] d \boldsymbol{r}=I^{\text {class. }}[p]+I^{\text {nclass. }}[p, \mathbf{k}, \phi],
\end{aligned}
$$

proportional to the system average kinetic energy

$$
T_{S}\left[\Psi_{\mathbf{k}}\right] \equiv\left\langle\Psi_{\mathbf{k}}|\hat{\mathrm{T}}| \Psi_{\mathbf{k}}\right\rangle=\frac{\hbar^{2}}{2 m} \sum_{i=1}^{N} \int\left|\nabla \varphi_{\boldsymbol{k}_{i}}(\boldsymbol{r})\right|^{2} d \boldsymbol{r}
$$

The latter consists of the "classical" density functional proposed by von Weizsäcker [24], depending solely upon the particle distribution,

$$
T^{\text {class. }}[\rho]=\frac{\hbar^{2}}{8 m} \int \frac{|\nabla \rho(\boldsymbol{r})|^{2}}{\rho(\boldsymbol{r})} d \boldsymbol{r},
$$

and the "non-classical", phase/current contribution,

$$
\begin{aligned}
T^{\text {nclass. }}[\rho, \phi] & =\frac{\hbar^{2}}{2 m N} \int \rho(\boldsymbol{r}) \sum_{l=1}^{N}\left(\nabla\left[\boldsymbol{k}_{l} \cdot \boldsymbol{f}(\boldsymbol{r})+\phi(\boldsymbol{r})\right]\right)^{2} d \boldsymbol{r}, \\
T\left[\Psi_{\mathbf{k}}\right] & =T^{\text {class. }}[\rho]+T^{\text {nclass. }}[\rho, \phi],
\end{aligned}
$$

related via Eq. (25) to the corresponding quantum information terms [6-8]:

$$
I\left[\Psi_{\mathbf{k}}\right]=I^{\text {class. }}[p]+I^{\text {nclass. }}[p, \phi] .
$$

The condition of the extremum Fisher information,

$$
\delta I\left[\Psi_{\mathbf{k}}\right] / \delta \phi(\boldsymbol{r})=\partial I^{\text {nclass. }}[p, \phi] / \partial \phi(\boldsymbol{r})=0,
$$

then determines the optimum phase that minimizes $I\left[\Psi_{\mathbf{k}}\right]$ and $I^{\text {nclass. }}[p, \phi]$ :

$$
\varphi_{\mathbf{k}}(\boldsymbol{r})=-\left(\frac{1}{N} \sum_{l=1}^{N} \boldsymbol{k}_{l}\right) \cdot \boldsymbol{f}(\boldsymbol{r}) \equiv-\boldsymbol{K}(\mathbf{k}) \cdot \boldsymbol{f}(\boldsymbol{r}) .
$$


The optimum phase is thus determined by the average "wave-number" vector $\boldsymbol{K}$ in $\Psi_{\boldsymbol{k}_{1}, \boldsymbol{k}_{1}, \ldots, \boldsymbol{k}_{N}} \equiv \Psi_{\mathbf{k}}$, for which the density of $I^{\text {nclass. }}$ exhibits the least structure ("order"), i.e., the maximum indeterminacy ("disorder"), reflected by the highest value of $S^{\text {nclass. }}$. The corresponding equidensity orbitals in this "thermodynamic" state,

$$
\left.\varphi_{\boldsymbol{k}_{l}}(\boldsymbol{r})=[\rho(\boldsymbol{r}) / N]^{1 / 2} \exp \left\{\mathrm{i}\left[\left(\boldsymbol{k}_{l}-\boldsymbol{K}\right) \cdot \boldsymbol{f}(\boldsymbol{r})\right]\right\} \equiv[\rho(\boldsymbol{r}) / N]^{1 / 2} \exp \left\{\mathrm{i}\left(\delta \boldsymbol{k}_{l}\right) \cdot \boldsymbol{f}(\boldsymbol{r})\right]\right\},
$$

determine the associated nonclassical contribution to the kinetic energy of electrons,

$$
\begin{aligned}
T^{n \text { class. }[\rho, \phi]} & =\frac{\hbar^{2}}{2 m} \int \frac{\rho(\boldsymbol{r})}{N} \sum_{l=1}^{N}\left(\nabla\left[\delta \boldsymbol{k}_{l} \cdot \boldsymbol{f}(\boldsymbol{r})\right]\right)^{2} d \boldsymbol{r} \\
& =\frac{\hbar^{2}}{2 m} \int p(\boldsymbol{r}) \sum_{l=1}^{N}\left(\nabla\left[\delta \boldsymbol{k}_{l} \cdot \boldsymbol{f}(\boldsymbol{r})\right]\right)^{2} d \boldsymbol{r},
\end{aligned}
$$

due to finite probability current

$$
\boldsymbol{j}(\boldsymbol{r})=\frac{\hbar}{m} \rho(\boldsymbol{r})\left(\frac{1}{N} \sum_{l=1}^{N} \delta \boldsymbol{k}_{l}\right) \cdot \nabla \boldsymbol{f}(\boldsymbol{r}) \equiv \frac{\hbar}{m} \rho(\boldsymbol{r}) \delta \boldsymbol{K} \nabla \cdot \boldsymbol{f}(\boldsymbol{r}) .
$$

The latter is seen to vanish only, when the corresponding deviation of the average reduced momentum vanishes: $\delta \boldsymbol{K}=\boldsymbol{0}$.

Consider next the equilibrium entropy/information principles [7,8], corresponding to the fixed ground-state electron density determined in the external search of Eq. (4): $\rho=\rho_{0}$. It involves the energy-constrained search for the optimum wave function of $N$-fermions, corresponding to the fixed external potential $v$ due to the system nuclei [see Eq. (2)]:

$$
\begin{aligned}
E\left[\Psi(N) \rightarrow \rho_{0}\right] & =E_{v}\left[\rho_{0}\right]=\int v(\boldsymbol{r}) \rho_{0}(\boldsymbol{r}) d \boldsymbol{r}+V_{e e}\left[\rho_{0}\right]+\inf _{\Psi \rightarrow \rho_{0}}\langle\Psi|\hat{\mathrm{T}}| \Psi\rangle \\
& =\int v(\boldsymbol{r}) \rho_{0}(\boldsymbol{r}) d \boldsymbol{r}+\inf _{\Psi \rightarrow \rho_{0}}\langle\Psi|\hat{\mathrm{F}}| \Psi\rangle
\end{aligned}
$$

One observes the presence of the Levy universal functional $F[\rho]$ [Eq. (2)] as the crucial (entropic) part of this EPI. Notice that the external potential and electron-repulsion energies are fixed by the frozen density constraint, so that the optimum state marks the infimum of the quantum Fisher measure of the information content in the optimum wave function.

Let us first examine the one-determinant approximation of the exact ground-state wave function, e.g., in the Kohn-Sham [7] theory, in which one assumes $\Psi_{0}(N)=$ $\Psi[N, v] \cong \Psi_{\mathbf{k}}(N)$. In this single-determinant case the optimum function $\Psi_{\mathbf{k}_{0}}\left(N ; \phi_{0}\right)$ results from the following Fisher EPI:

$$
\inf _{\Psi \rightarrow \rho_{0}} I[\Psi]=I^{\text {class. }}\left[p_{0}\right]+\inf _{\Psi \rightarrow \rho_{0}} I^{\text {nclass. }}\left[p_{0}, \mathbf{k}, \phi\right] \Rightarrow \Psi_{\mathbf{k}_{0}}\left(N ; \phi_{0}\right)
$$


In the Harriman-type construction of the Fisher measure of information the optimum ground state is thus determined by the optimum values of the "wave-number" vectors $\mathbf{k}=\mathbf{k}_{0}=\left\{\boldsymbol{k}_{l}^{0}\right\}$ and the associated optimum phase function [Eq. (32)]:

$$
\phi(\boldsymbol{r})=\phi_{0}(\boldsymbol{r})=-\left(\frac{1}{N} \sum_{l=1}^{N} \boldsymbol{k}_{l}^{0}\right) \cdot \boldsymbol{f}_{0}(\boldsymbol{r}) \equiv-\boldsymbol{K}\left(\mathbf{k}_{0}\right) \cdot \boldsymbol{f}_{0}(\boldsymbol{r}), \quad \boldsymbol{f}_{0} \equiv \boldsymbol{f}\left[\rho_{0}\right]
$$

Together they uniquely specify $N$ singly-occupied spin-orbitals of the "thermodynamic" Slater determinant $\Psi_{\mathbf{k}_{0}}\left(\boldsymbol{r} ; \phi_{0}\right) \equiv \Psi^{(0)}(\boldsymbol{r})$. The minimum Fisher-information principle of Eq. (36) thus involves a search for the optimum equidensity orbitals of $N$ electrons in the ground-state electron distribution $\rho_{0}$, determined by their optimum "wave-number" vectors $\mathbf{k}_{0}$ and the associated phase of Eq. (38).

As argued elsewhere [7,8] both the classical and non-classical parts of the densities per electron of the quantum Fisher and Shannon information measures [Eqs. (22-25)] are mutually related:

$$
\begin{aligned}
I^{\text {class. }}(\boldsymbol{r}) & =[\nabla \ln p(\boldsymbol{r})]^{2}=\left[\nabla S^{\text {class. }}(\boldsymbol{r})\right]^{2} \text { and } \\
I^{\text {nclass. }}(\boldsymbol{r}) & =\left(\frac{2 m \boldsymbol{j}(\boldsymbol{r})}{\hbar p(\boldsymbol{r})}\right)^{2} \equiv\left[\nabla S^{\text {nclass. }}(\boldsymbol{r})\right]^{2} .
\end{aligned}
$$

One further observes that for the fixed electron distribution in Eq. (36) only the nonclassical components depend upon the "wave-number" vectors $\mathbf{k}$ and the phase function $\phi(r)$, which together determine the resultant phase in Harriman's construction, to be optimized in the associated entropic principle. This infimum of the Fisher measure for $p=p_{0}$ implies the least average gradients of the wave function orbitals [see Eq. (25)], and hence their lowest degree of structure (order). This generates the highest admissible degree of the wave-function indeterminacy (disorder), as marked by the supremum of the quantum Shannon entropy:

$$
\sup _{\Psi \rightarrow \rho_{0}} S[\Psi]=S^{\text {class. }}\left[p_{0}\right]+\sup _{\Psi \rightarrow \rho_{0}} S^{\text {nclass. }}\left[p_{0}, \mathbf{k}, \phi\right] \Rightarrow \Psi_{\mathbf{k}_{0}}\left(N ; \phi_{0}\right) .
$$

Therefore, the two generalized measures of the information content in complex wave-functions of the Harriman-type are complementary in character: the groundstate density/energy constrained EPI of the lowest quantum Fisher information is synonymous with the related constrained EPI of the highest quantum Shannon entropy. This is reminiscent of the complementary equilibrium criteria of the minimum energy and maximum entropy in phenomenological thermodynamics [16].

Finally, let us briefly examine a general case of a trial wave function $\Psi_{0}(N)$ of an $N$-electron molecular system, corresponding to the prescribed electron density $\rho=\rho_{0}$. In the pure-state representation the ground state $\Psi_{0}(N)$ can be expanded into the complete set of $N$-electron determinants of Eqs. (16) and (19), containing a common modulus (density) factor of Eq. (18), 


$$
R_{0}(N)=\prod_{k=1}^{N}\left(\frac{\rho_{0}\left(\boldsymbol{r}_{k}\right)}{N}\right)^{1 / 2}
$$

and the phase factor $\Phi(N)$ of Eq. (15), in the continuous Configuration Interaction (CI) type expansion,

$$
\Psi_{0}(N)=\int C_{0}(\mathbf{k}) \Psi_{\mathbf{k}}(N) d \mathbf{k}
$$

The expectation value $\langle A\rangle^{\mathrm{CI}}$ of the physical quantity $A$ represented by operator $\hat{\mathrm{A}}$ is then given by the familiar expression

$$
\langle A\rangle^{\mathrm{CI}}=\iint C_{0}^{*}(\mathbf{k}) C_{0}\left(\mathbf{k}^{\prime}\right) A\left(\mathbf{k}, \mathbf{k}^{\prime}\right) d \mathbf{k} d \mathbf{k}^{\prime}, \quad A\left(\mathbf{k}, \mathbf{k}^{\prime}\right)=\left\langle\Psi_{\mathbf{k}}|\hat{\mathrm{A}}| \Psi_{\mathbf{k}}^{\prime}\right\rangle .
$$

The linear variational parameters $\left\{C(\mathbf{k})=\left\langle\Psi_{\mathbf{k}} \mid \Psi\right\rangle\right\}$ also generate to the conditional probabilities

$$
\left\{P_{0}\left(\Psi_{\mathbf{k}} \mid \Psi_{0}\right)=\left|C_{0}(\mathbf{k})\right|^{2} \equiv P_{0}(\mathbf{k})\right\}, \quad \int P_{0}(\mathbf{k}) d \mathbf{k}=1
$$

of the density constrained determinants in the ensemble defined by the density operator

$$
\hat{\mathrm{D}}_{0}(N)=\int d \mathbf{k}\left|\Psi_{\mathbf{k}}\right\rangle P_{0}\left(\Psi_{\mathbf{k}} \mid \Psi_{0}\right)\left\langle\Psi_{\mathbf{k}}\right|
$$

The representative ensemble-average values of the physical properties in such a mixed CI state then read $A^{\mathrm{CI}}=\int P_{0}(\mathbf{k}) A(\mathbf{k}, \mathbf{k}) d \mathbf{k}$, e.g.,

$$
\begin{array}{rlrl}
\rho^{\mathrm{CI}} & =\rho_{0} \int P_{0}(\mathbf{k}) d \mathbf{k}=\rho_{0}, & \boldsymbol{j}^{\mathrm{CI}}(\boldsymbol{r})=\int P_{0}(\mathbf{k}) \boldsymbol{j}_{\mathbf{k}}(\boldsymbol{r}) d \mathbf{k}, \\
\boldsymbol{K}^{\mathrm{CI}}=\int P_{0}(\mathbf{k}) \mathbf{K}(\mathbf{k}) d \mathbf{k}, & \phi_{\mathbf{k}_{0}}^{\mathrm{CI}}(\boldsymbol{r})=-\boldsymbol{K}^{\mathrm{CI}} \cdot \boldsymbol{f}_{0}(\boldsymbol{r}), \text { etc. }
\end{array}
$$

\section{Conclusion}

It has been argued, using the $N$-electron Harriman-Zumbach-Maschke construction of the antisymmetric wave function yielding the prescribed electron density, that the optimum equilibrium ("thermodynamic") state of $N$ electrons, resulting from the complementary Fisher or Shannon EPI principles, generally differs from the (nondegenerate) ground state of the electronic Schrödinger equation, the lowest eigenstate of the system electronic Hamiltonian, in which the probability current identically vanishes. Indeed, for a variational density $\rho$ the optimum (position-dependent) phases of the Harriman equidensity orbitals in such an equilibrium state generally imply the nonvanishing phase-gradients of the equidensity orbitals, and hence also a presence of the finite probability current of the system $N$ electrons, for the fixed electron distribution, i.e., the conserved electronic energy. This qualitatively confirms the main conclusion following from previous one-electron analysis [8]. For a more detailed exploration of 
the molecular equilibria using the nonclassical information measures the reader is also referred to a recent analysis [25].

The reduced momenta $\mathbf{k}$, which determine the phase factors of the equidensity orbitals, provide the continuous state-variables generating the crucial density of determinantal states, which is essential in any adequate, thermodynamic-like perspective on the vertical (fixed-density) variations of the system wavefunction.

Notice, however, that for the exact density $\rho=\rho_{0}$ of the nondegenerate groundstate the best single-determinant $\Psi_{\mathbf{k}_{0}}\left(N ; \phi_{0}\right)$ approximation of the optimum thermodynamic state should recover the Slater determinant of the Kohn-Sham [12] quality. Then for $K=K_{0}$ and hence the vanishing current one indeed realistically approximates the true ground-state of the $N$-electron system in question: $\Psi[N, v] \cong \Psi_{\mathbf{k}_{0}}\left(N ; \phi_{0}\right)$. This is in accordance with the HK theorem, that in such a case the electron density uniquely identifies the system ground-state.

One further observes, that for the exact ground-state density $\rho=\rho_{0}$, i.e., $f=f\left[\rho_{0}\right]=f_{0}, \mathbf{k}=\mathbf{k}_{0}$, and hence $K^{\mathrm{CI}}=K_{0}$, the associated displacement $\delta K^{\mathrm{CI}}$ in the average reduced momentum identically vanishes in the equilibrium state, for $\phi=\phi_{0}, \delta \boldsymbol{K}^{\mathrm{CI}}=\boldsymbol{0}$. This implies an exactly vanishing probability current of Eq. (33), and hence the exact ground state of the system in question: $\Psi_{0}(N)=\Psi[N, v]$. This prediction again follows the first HK theorem, that the exact electron distribution of the nondegenerate ground-state uniquely determines the system wave function: $\Psi_{0}=\Psi_{0}\left[\rho_{0}\right]$.

Open Access This article is distributed under the terms of the Creative Commons Attribution License which permits any use, distribution, and reproduction in any medium, provided the original author(s) and the source are credited.

\section{References}

1. R.A. Fisher, Proc. Camb. Phil. Soc. 22, 700 (1925)

2. C.E. Shannon, Bell System Tech. J. 27, 379, 623 (1948)

3. B.R. Frieden, Physics from Fisher Information: A Unification (Cambridge University Press, Cambridge, 1998)

4. M.J.W. Hall, Phys. Rev. A 62, 012107 (2000)

5. A. Nagy, Chem. Phys. Lett. 449, 212 (2007)

6. R.F. Nalewajski, Int. J. Quantum Chem. 108, 2230 (2008)

7. R.F. Nalewajski, in Foundations of Chemistry: Quantum Theory of Atoms and Molecules, ed. by C. Matta (in press)

8. R.F. Nalewajski, Entropic representation in the theory of molecular electronic structure. J. Math. Chem. (in press)

9. R.F. Nalewajski, Information Theory of Molecular Systems (Elsevier, Amsterdam, 2006)

10. M. Levy, Proc. Natl. Acad. Sci. USA 76, 6062 (1979)

11. P. Hohenberg, W. Kohn, Phys. Rev. 136B, 864 (1964)

12. W. Kohn, L.J. Sham, Phys. Rev. 140A, 1133 (1965)

13. R.G. Parr, W. Yang, Density Functional Theory of Atoms and Molecules (Oxford, New York, 1989)

14. R.M. Dreizler, E.K.U. Gross, Density Functional Theory: An Introduction to the Quantum Many-Body Problem (Springer, Berlin, 1990)

15. R.F. Nalewajski, ed. Density Functional Theory I-IV, Topics in Current Chemistry, vols. 180-183 (Springer, Heidelberg, 1996)

16. H.B. Callen, Thermodynamics: An Introduction to the Physical Theories of the Equilibrium Thermostatics and Irreversible Thermodynamics (Wiley, New York, 1960) 
17. J.E. Harriman, Phys. Rev. A 24, 680 (1981)

18. W. Macke, Ann. Phys. Leipzig 17, 1 (1955)

19. T.L. Gilbert, Phys. Rev. B 12, 2111 (1975)

20. N.H. March, Phys. Rev. A 26, 1845 (1982)

21. G. Zumbach, K. Maschke, Phys. Rev. A 28, 544. Erratum, Phys. Rev. A 29, 1585 (1984)

22. R.F. Nalewajski, in Recent Advances in Density Functional Methods, part III, ed. by V. Barrone, A. Bencini, P. Fantucci (World Scientific, Singapore 2002), p. 257

23. A. Nagy, S.B. Liu, Phys. Lett. A 372, 1654 (2008)

24. C.F. von Weizsäcker, Z. Phys. 96, 431 (1935)

25. R.F. Nalewajski, Ann. Phys. Leipzig (submitted) 